\title{
Sports Athletes Real-time Analysis Model based on Wearable Devices and Intelligent Detection System
}

\author{
Jianqiang Feng ${ }^{1}$, Yuanxiang Chen ${ }^{2}$ \\ ${ }^{1}$ Xi'an University of technology, Xi'an, 710048, China; \\ ${ }^{2}$ Xi'an Peihua University, Xi'an, 710065, China.
}

Keywords: Athletes, Sports, Intelligent Detection, Wearable Devices, Real-time, Model Form.

\begin{abstract}
In this paper, we design and implement the sports athlete real-time analysis model based on wearable devices and intelligent detection system. Because of the wearable computer man-machine interactive mode and normal computer differences in way of the human-computer interaction, led to a wearable computer on the hardware structure is very different with traditional computer. In this paper, the wearable computer is discussed in detail two different hardware structures adopted by the way: the centralized and the distributed structure. Centralized structure is developed based on the traditional computer, suitable for rapid development and the development of wearable computer. With this basic assistance, we later analyze the integration of analysis model and wearable devices that will enhance the performance of the detection system which is meaningful.
\end{abstract}

\section{Introduction}

Use basic remote microcomputer and electronic technology, the athletes in physical training, on-site analysis of physiological load response data processing, and the results timely feedback to the coaches and sports science and technology personnel, for the practical implementation of the coaches insight into this session, an objective assessment of the quality of class, the next training plan scientifically, in order to improve the general efficiency of physical training has important theoretical significance and practical application value. Scientific training monitoring including the diagnosis and monitoring of body function, sport techniques in diagnosis and monitoring, diagnosis and monitoring the content of the aspects of psychological state as monitoring is carried out on the track and field athletes training, with the physiological and biochemical indexes to evaluate the functional status of athletes [1-2].

With three-dimensional photography for technology evaluation and improvement of the athletes, such as using standardized questionnaires and advantages and disadvantages listed methods to test the athletes' mental state, etc. the scientific training monitoring is to use the theory and method of relevant subject, measurement and evaluation of related index in the process of the training, so as to realize the optimization of training process. According to the literature review, the monitoring points could be generally summarized as the follows. (1) Blood urea. Blood urea is caused by protein and amino acid metabolism of urea, its function with human movement state, sports load and fatigue state diagnosis. (2) Hemoglobin. The main function is as the red blood cell hemoglobin to transport oxygen carrier, at the same time have the effect that maintain acid-base balance in the body. But it is not the more the better, it can lead to a rise in blood viscosity, blood flow velocity slowed that adverse to the body. (3) Sphygmus. Record the pulse of the times a day, and draw a graph, if the curve can be stable or falling, that function is in good condition, sports load is appropriate; On the other hand, it may be caused by load inappropriate or sick. (4) Blood pressure. Blood pressure is within the artery blood in the vessel wall lateral pressure, it is due to the interaction between ventricular ejection and peripheral resistance.

To assist the coaches for better evaluation of the athletes, in this paper, we conduct research on the sports athlete real-time analysis model based on wearable devices and intelligent detection system. Sports in our country a lot of practice in this regard, diving is ready to use the computer 
aided training system to train, weightlifting and enhance the accuracy of player movement using the decomposition of action system, fire project is adopted the laser system, tracking ballistic direction and help optimize the shooting angle, with understanding of the relationship between the angle of the differences and precision, and can be used to adjust in a timely manner. Bicycle project also configured the high-speed mobile camera to real-time capture the athlete action details will have a quantitative analysis of action and get some useful data, and then can improve the athletes' games way and optimize the physical movement in detail. In the figure one, we illustrate the sample interface.

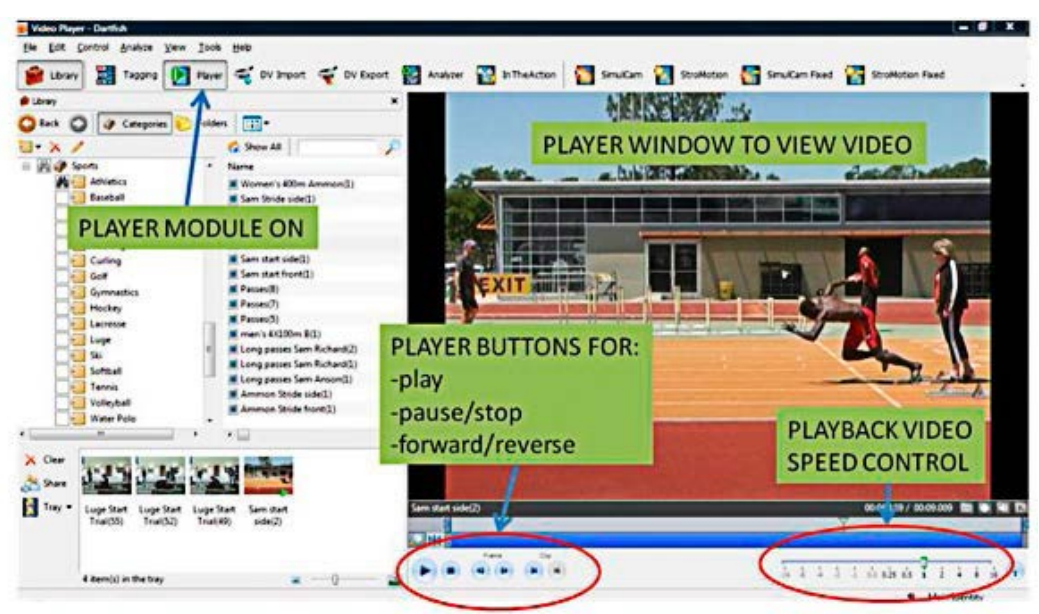

Fig. 1 The Sample Interface of the Intelligent Detection System

\section{Our Designed System}

The Wearable Devices. At present, the development and the development of wearable computer is still in its infancy groping stage, more prototype and product uses is the relevant miniaturization of the electronic equipment, combining with some wearable technology. Although the wearable computer from seamless, the best combination and coordination between the man-machine idea is a long way, but it is the basic capability of the wearable computer some attributes and functions, in people's daily life, the large complex equipment maintenance and overhaul, medical, geological exploration and the military fields are showed its potential application value.

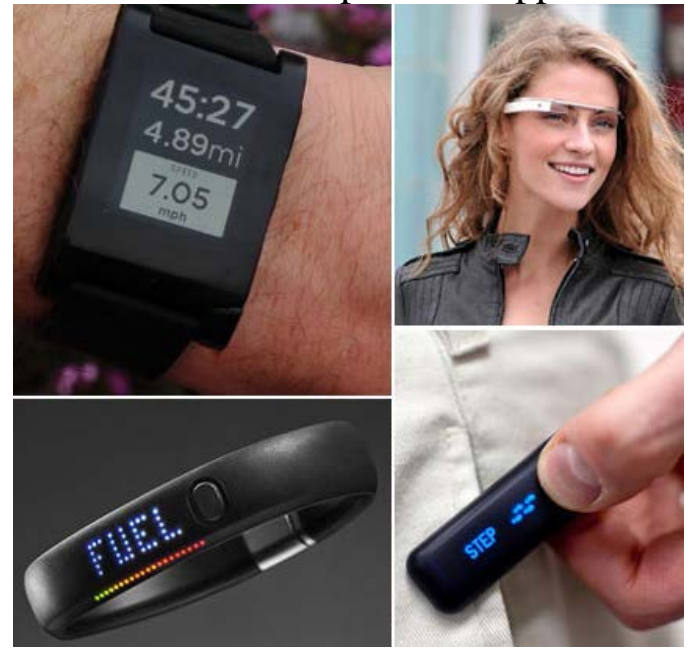

Fig. 2 The Demonstration of the Wearable Devices

At present, the general categories of the wearable devices could be summarized as follows. (1) Centralized wearable computer. Centralized wearable computer is developed on the basic basis of the existing computer that is the product of the computer technology combined with wearable technology, inherited the many mature techniques of the computer. Centralized wearable computer is currently the research institutions for wearable computer prototype is developed widely adopted by the project, its biggest advantage is the ability to adopt the basic COST product on the basis of the existing computer technology. (2) Distributed wearable computer. Distributed wearable 
computer in order to overcome the centralized wearable computer because of size and weight limit inconvenience to users that will the machine according to different separation of main functional modules, to reduce the volume and weight of each specific device, and connecting with the distribution of the man-machine engineering principle of each module in the human body is suitable for wear parts [3-4].

To provide the users with a wearable computer computing services anytime and anywhere, it to the user wear content for the system support, weaken the traditional form of computer. The development trend of the wearable computer is gradually weakening the concept of the machine, no longer in the traditional form of machine, but machine can be incorporated into everyday people wear accessories.

Sports Athletes and the Computer.Modern high level of general competitive sports training has profound relies on the multi-disciplinary high-tech comprehensive protection. Modern sports training from general physical quality and core training principle and tactics of research into the biochemistry, physiology, biomechanics, psychology, genetics and even free radical theory, the theoretical study of the doctrine of genetic traits, that need on sports level closely related mechanism of the formation of multi-level multi-dimensional thinking system [5-6].

A function of the training of the high efficiency and fast strain monitoring and regulation of basic computer information management system design should achieve the following three goals. (1) The monitoring that embodies the unity of the monitoring information and means of feedback, conducive to scientific survey in approach and its effect, analysis, and strain. (2) Macro description, reflects the multidisciplinary multidimensional, to be clear at a glance, for the coaches athletes and administrative leadership to master the general situation of the whole project progress. (3) Microscopic record, that reflects drill-down, elements, the detailed for the coaches and the scientific researchers on the athletes data in-depth mastery and comprehensive analysis.

Now to the training of the athletes most function monitoring and regulating the management of the traditional method, test indicators of athletes, grades, the measures are still using artificial records, the artificial sorting, retrieval query. Therefore it is necessary to establish a high efficiency and fast strain training function monitoring and regulation of computer information management system.

Intelligent Detection Devices and Hardware. Smart hardware is a basic concept of science and the technology after the smartphone, through the combination of hardware and software, modification or development to the traditional equipment, new equipment, then let it has function of the intelligent that have the ability to connect, intelligent, hardware implementation of Internet service load.

With "data mobile technology" intelligent data test management system, not only to be able to test data to the data and histogram display, but also expressed as a dynamic display mode curve detection data. Through this kind of display mode, the operator can visually dynamic change trend of observed data, and learned that in the certain period of time the data change process, at the beginning of the equipment to start this way of testing data show that is particularly important.

Smart hardware has been extend from wearable devices to smart TV, smart home, intelligent car, medical health, the intelligent toys, robot, etc. Typical intelligent hardware including GoogleGlass, Samsung, Gear, wheat open cups, plump hand ring, Tesla, Letv TV, etc. Smart hardware can better in life, to form a complete set of ecosystem [7].

On the other hand, the intelligent hardware only one monomer machine, form is not the power of the polymerization. On the development of intelligent hardware, should consciously with the aid of the existing platform, from the statistical analysis to access hardware and firmware upgrades to social comprehensive docking, the force and improve the development efficiency.

Sports Athletes Real-time Analysis Model. Health management is a kind of produced in the United States, popular in the world of health concept and method, the current has been increasingly brought to the attention of countries all over the world and the different groups of health management practice, has increasingly become an effective means of prevention and treatment of various diseases. Health management in traditional medicine, management science and the 
computer science and other multi-disciplinary knowledge which is to the individual or group health to conduct a comprehensive monitoring, analysis and assessment, provide health advice and guidance, and the whole process of the risk factors for health intervention [8].

Understand the athletes' mental state, on one hand may stimulate the athlete exercise enthusiasm, promote the development of their intelligence and the perfection of personality, to help the athletes to establish correct training motivation; On the other hand adjust the training plan provides the basis for the coaches which could be separated into the listed aspects. (1) Psychological indicators including the regulators and the reaction time, the two thresholds as the flicker fusion frequency. There are five kinds of commonly used the self-report scale including mental state scale, horse tesla grams of mental fatigue scale, the athlete mental exhaustion scale, athlete psychological burnout questionnaire and the athletes training condition monitoring scale. Reaction time, with the two thresholds and flicker fusion frequency test of three kinds of simple and feasible, and the quantization degree is higher, but need serious athletes to cooperate. (2) Intelligence test, also known as common ability test or general ability test, is a measure of the level of intelligence by means of test method. At present, the most commonly used intelligence tests have to sport psychology adult intelligence scale and raven standard reasoning test. (3) Confidence can help the weak against the strong. The measurement method of confidence has a lot of features that can generally be listed by the self-report scale method and the advantages and the disadvantages of methods for measuring.

\section{Summary}

In this paper, we design and implement the sports athlete real-time analysis model based on wearable devices and intelligent detection system. Intelligent wearable devices, is the partial function of mobile devices can be incorporated into the traditional wearable items. At present, the concept of wearable is gradually inspire all kinds of creative application, almost all equipment related to the human body can plug in the wings of the Internet, has become the darling of the capital and industry. At the same time, the development of wearable devices will also change people's cultural consumption patterns. All the successful platforms need to be a strong support system to support the idea, and then realize the needs of users, to retain users, and further attract basic users to create a sustainable platform for the positive feedback effect. Under this background, we propose the sports athlete real-time analysis model with the assistance of the intelligent detection system that will be innovative.

\section{References}

[1] Jia, Wenzhao, et al. "Electrochemical tattoo biosensors for real-time noninvasive lactate monitoring in human perspiration." Analytical chemistry 85.14 (2013): 6553-6560.

[2] King, D., et al. "The influence of head impact threshold for reporting data in contact and collision sports: systematic review and original data analysis." Sports medicine (2015): 1-19.

[3] McAllister, Thomas W., et al. "Effect of head impacts on diffusivity measures in a cohort of collegiate contact sport athletes." Neurology 82.1 (2014): 63-69.

[4] van den Bogert, Antonie J., et al. "A real-time system for biomechanical analysis of human movement and muscle function." Medical \& biological engineering \& computing 51.10 (2013).

[5] McAllister, Thomas W., et al. "Effect of head impacts on diffusivity measures in a cohort of collegiate contact sport athletes." Neurology 82.1 (2014): 63-69.

[6] Dwyer, Dan B., and Tim J. Gabbett. "Global positioning system data analysis: Velocity ranges and a new definition of sprinting for field sport athletes." The Journal of Strength \& Conditioning Research 26.3 (2012): 818-824.

[7] Harrison, Craig B., et al. "Development of Aerobic Fitness in Young Team Sport Athletes. 